Célia Landmann Szwarcwald 1

\title{
Estimação da mortalidade infantil no Brasil: - que dizem as informações sobre óbitos e nascimentos do Ministério da Saúde?
}

\author{
Infant mortality estimation in Brazil: \\ what do Ministry of Health data on deaths \\ and live births say?
}

Maria do Carmo Leal 2

Carla Lourenço Tavares de Andrade 1

Paulo Roberto Borges de Souza Jr. 1

\footnotetext{
1 Departamento de Informações em Saúde Centro de Informação Científica e Tecnológica, Fundação Oswaldo Cruz. Av. Brasil 4365, RJ 21045-900, Brasil. celials@cict.fiocruz.br pborges1@brfree.com.br 2 Departamento de Epidemiologia e Métodos Quantitativos em Saúde, Escola Nacional de Saúde Pública, Fundação Oswaldo Cruz. Rua Leopoldo Bulhões 1480, Rio de Janeiro, $R J$ 21041-210, Brasil. duca@ensp.fiocruz.br
}

\begin{abstract}
A methodological approach to infant mortality estimation in Brazil based on vital information provided by Ministry of Health systems is presented. The study evaluated the available data to establish criteria for identifying municipalities with serious data deficiencies, proposing an adequacy index. All municipalities were classified in strata according to geographic region and adequacy of information. To estimate infant mortality by macro-geographic region, in 1998, direct calculation was performed in strata with adequate information. The United Nations model was used in the other geographic strata. The Brazilian North presented the most deficient information, with $63 \%$ of the municipalities presenting inadequate reporting (35\% of the regional population), followed by the Northeast (29\% of the population). In the South, only $1 \%$ of the population showed inadequate information. For the whole country, $12 \%$ of the population presented serious problems in completeness of death reports. The adequacy index varied from -28\% in Maranhão to 94\% in Rio de Janeiro. The infant mortality rate was estimated in the interval 30.7-32.6 per 1,000 live births and the completeness of infant deaths from 61.8\% to 65.6\%.

Key words Infant Mortality; Mortality Rate; Information Systems; Underregistration
\end{abstract}

Resumo Apresenta-se uma proposta metodológica para a estimação da mortalidade infantil, no Brasil, mediante a utilização das informações do Ministério da Saúde. O estudo consistiu em estabelecer critérios para identificar municípios com limitações nas informações, propondo-se um índice para sintetizar a adequação. Os municípios foram, então, agregados em estratos, por Grande Região, de acordo com a categoria de adequação das informações disponíveis. Para estimar a mortalidade infantil por região, no ano de 1998, utilizou-se o cálculo direto nos estratos com adequação das informações e o modelo das Nações Unidas nos demais. Verificou-se que a Região Norte é a que possui as maiores deficiências, com 63\% dos municípios com notificação inadequada (35\% da população da região), seguida da Nordeste (29\% da população). Já na Região Sul, somente 1\% da população apresenta grande precariedade dos dados de óbitos. Para a totalidade do Brasil, 12\% da população apresenta grandes deficiências nas informações. O índice de adequação variou de -28\%, no Maranhão, a 94\%, no Rio de Janeiro. A mortalidade infantil foi estimada no intervalo 30,7-32,6 por 1.000 nascidos vivos e a cobertura de óbitos infantis, de $61,8 \%$ a $65,6 \%$.

Palavras-chave Mortalidade Infantil; Coeficiente de Mortalidade; Sistemas de Informação; Sub-Registro 


\section{Introdução}

No Brasil, a coleta, a apuração e a divulgação dos eventos vitais são de responsabilidade da Fundação Instituto Brasileiro de Geografia e Estatística (IBGE). Desde 1974, as estatísticas de registro de óbitos e nascimentos têm sido divulgadas, anualmente, por meio da publicação Estatísticas do Registro Civil (IBGE, 1998).

O Sistema de Informações sobre Mortalidade (SIM) do Ministério da Saúde (MS), constitui-se em uma fonte alternativa de dados de óbitos no país. Criado em 1976, a partir da implantação do modelo padronizado da declaração de óbito (DO) em todo o território nacional, o SIM é justificado não só para o atendimento de exigências legais, mas tem o objetivo principal de fornecer subsídios para traçar o perfil de mortalidade no país (MS, 1982).

Apesar da disponibilidade de dois sistemas de informações sobre óbitos em nível nacional, o do Registro Civil e o SIM, um dos grandes problemas que ainda limitam as análises de mortalidade no Brasil é o sub-registro de óbitos, que tem magnitude expressiva, sobretudo, nas regiões Norte e Nordeste, com predomínio entre as crianças com menos de um ano de idade. O sub-registro de óbitos diz respeito, principalmente, à ocorrência de sepultamentos sem a exigência da certidão, nos denominados cemitérios clandestinos, sendo associado à pobreza e prevalente na área rural (Mello-Jorge, 1983, 1987).

No que se refere às informações referentes aos nascimentos, em 1990, o MS implantou o Sistema de Informação sobre Nascidos Vivos (SINASC). Esse sistema tem base na declaração de nascimento, documento cuja emissão é considerada obrigatória no serviço de saúde onde ocorreu o parto. Diferentemente do sistema de nascimentos do Registro Civil, cujo objetivo principal é a contagem do número de registros de nascimentos, o SINASC tem como propósito caracterizar as condições de nascimento (Mello-Jorge et al., 1996). Embora seja perceptível que a cobertura do SINASC esteja crescendo e que a qualidade da informação venha melhorando desde a sua implantação, sabe-se que a cobertura do sistema ainda não é completa. A comparação entre o número de nascimentos ocorridos nos hospitais do Sistema Único de Saúde (SUS) e os declarados no SINASC, mostra ainda deficiências na cobertura do SINASC em alguns estados do país, embora de menor magnitude que as apresentadas pelo SIM (Schramm \& Szwarcwald, 2000).

Diante das limitações das fontes de informações, o IBGE, que é o órgão responsável por fornecer a estimativa da mortalidade infantil no Brasil, tem recorrido a técnicas demográficas, ditas de mensuração indireta, para substituir as estimativas clássicas (IBGE, 2001). Tais técnicas são baseadas em dados de entrevistas domiciliares realizadas por ocasião dos Censos Demográficos ou das Pesquisas Nacionais de Amostra por Domicílio (PNAD).

$\mathrm{Na}$ ausência de informações completas no nível nacional, o MS tem optado por divulgar as estimativas da mortalidade infantil realizadas pelo IBGE por métodos demográficos de mensuração indireta. Entretanto, a comparação dos resultados obtidos por cálculo direto com aqueles obtidos por técnicas de mensuração indireta, em estados que, reconhecidamente, têm sistemas de mortalidade com cobertura satisfatória das informações de registro, tem mostrado inconsistências relevantes e superestimativas da mortalidade infantil no nível nacional.

A Rede Interagencial de Informações para a Saúde (RIPSA), criada por iniciativa conjunta do MS e da Organização Pan-Americana da Saúde (OPAS), divulga, anualmente, Indicadores e Dados Básicos de Saúde (IDB), incluindo-se entre eles a mortalidade infantil, segundo as Unidades da Federação (UF). Em iniciativa pioneira no Brasil, a RIPSA, via IDB-2000, já vem divulgando taxas de mortalidade infantil, calculadas pelo método direto, em sete dos estados brasileiros, onde, por critérios estabelecidos por integrantes da RIPSA, a cobertura das informações de registro é considerada adequada. Nos demais estados, são divulgadas as estimativas desse indicador obtidas por mensuração indireta (DATASUS, 2001). Comparando-se as estimativas do IBGE com as divulgadas pela RIPSA, referentes ao ano de 1998, verifica-se que a estimativa da RIPSA para a totalidade do Brasil, de 33,1 por 1.000 nascidos vivos (NV), é $10 \%$ menor do que a estimativa fornecida pelo IBGE, de 36,1 por $1.000 \mathrm{NV}$, baseada na estimação indireta para todas as UF (IBGE, 2001).

No que diz respeito às estimativas no nível municipal, por solicitação do MS, Simões (1999) elaborou estimativas das taxas de mortalidade infantil para todos os municípios brasileiros com mais de 80 mil habitantes, referentes ao ano de 1998, que estão sendo apresentadas na página do Departamento de Informática do SUS (DATASUS) na Internet, como estimativas oficiais da mortalidade infantil nessas localidades. Entretanto, as taxas foram estimadas por mensuração indireta, indistintamente para todos os municípios considerados, incluindo aqueles em que o SIM e o SINASC se mostram com cobertura completa e de quali- 
dade adequada. Devido ao fato das taxas apresentadas estarem superestimando os valores do coeficiente de mortalidade infantil, em decorrência do procedimento metodológico empregado, as estimativas têm causado reações negativas e de desestímulo aos profissionais de saúde que vêm desenvolvendo ações especificamente dirigidas à diminuição dos óbitos infantis (ABRASCO, 2001).

Recentemente, o MS tem estimulado o debate sobre a forma mais adequada de estimar a mortalidade infantil no país. O presente trabalho tem por objetivo propor uma proposta metodológica para a estimação deste indicador mediante a utilização das informações do SIM e do SINASC. Parte-se do princípio de que nos locais para os quais a cobertura dos sistemas de informação é adequada, a mortalidade infantil deve ser calculada de forma direta.

\section{Metodologia}

A primeira parte do trabalho consistiu em elaborar uma análise das informações do SIM e do SINASC. Para todos os municípios brasileiros, foram coletados dados sobre óbitos, nascimentos e população, tendo como fonte de informações a página do DATASUS (2001), utilizada pelo MS para divulgação de suas informações. A análise possibilitou avaliar as estimativas do IBGE obtidas por mensuração indireta e elaborar critérios para classificar os municípios quanto à adequação das informações disponíveis.

Foram construídos os seguintes indicadores:

\section{a) Coeficiente geral de mortalidade padronizado por idade}

O coeficiente geral de mortalidade (CGM) é definido pelo número total de óbitos, por mil habitantes, em uma determinada população, em um determinado ano. Por ser influenciado pela estrutura etária da população, para fins comparativos, optou-se por utilizar o coeficiente geral de mortalidade padronizado por idade ao invés do coeficiente bruto (Kahn \& Sempos, 1989). No presente trabalho, foi considerada como população-padrão a do Estado do Rio de Janeiro, por ser o estado com a maior proporção de idosos do Brasil (DATASUS, 2001).

O coeficiente de mortalidade padronizado por idade pode ser utilizado para identificar falhas na cobertura das informações de registro de óbitos. Geralmente varia entre 7 e 10 por 1.000 habitantes. Valores menores do que 4 por 1.000 habitantes indicam grande precariedade na cobertura das informações de mortalidade (Becker, 1991).

\section{b) Desvio médio do coeficiente geral de mortalidade no período 1996-1998}

Sabendo-se que não há grandes alterações no coeficiente geral de mortalidade de uma população em um período curto de três anos, este indicador pode ser utilizado para avaliar a regularidade das informações de óbitos.

Na presente análise, foram calculados, primeiramente, os coeficientes gerais de mortalidade para os municípios brasileiros, referentes aos anos de 1996, 1997 e 1998. A seguir, foi calculada a média aritmética dos coeficientes. $\mathrm{O}$ desvio médio foi, então, definido como a média aritmética dos valores absolutos dos desvios do CGM, em cada ano, em relação à média. Matematicamente, é expresso por:

$$
D M C G M=\frac{|C G M 96-C G M M E D|+|C G M 97-C G M M E D|+|C G M 98+C G M M E D|}{3 x \text { CGMMED }}
$$

onde

$$
C G M M E D=\frac{C G M 96+C G M 97+C G M 98}{3}
$$

Valores maiores do que $10 \%$ foram considerados críticos.

\section{c) Taxa de natalidade}

A taxa de natalidade é calculada pela razão entre o número de nascidos vivos e a população total em um determinado ano. Este indicador foi utilizado para apreciação das informações do SINASC entre as unidades geográficas sob análise. Valor muito baixo indica subenumeração de nascidos vivos enquanto valor muito alto aponta para invasão de registros de nascimentos. No caso do Brasil, 16 por 1.000 habitantes foi considerado como o limite crítico inferior. Entretanto, a larga amplitude de variação da taxa de natalidade restringiu a escolha de um limite crítico superior, em nível nacional.

\section{d) Desvio médio da taxa de natalidade}

Da mesma forma que o coeficiente geral de mortalidade, é esperado que não haja grandes alterações na taxa de natalidade em um período de três anos. Expressando a flutuação no número de nascidos vivos informados, esse indicador é construído de maneira semelhante ao desvio médio do CGM. Analogamente, para calcular o desvio médio da taxa de natali- 
dade, foram considerados os valores absolutos dos desvios da taxas brutas de natalidade em cada ano em relação à média das taxas de natalidade no período 1996-1998. Valores superiores a $10 \%$ foram, igualmente, considerados críticos.

\section{e) Proporção de óbitos em menores} de um ano sem definição da causa básica

A proporção de óbitos classificados na rubrica "sintomas, sinais e achados anormais de exames clínicos e de laboratório não classificados em outra parte" representa a fração das mortes em que não houve definição da causa básica de óbito entre as crianças menores de um ano. $\mathrm{O}$ uso desse indicador na presente análise justifica-se por expressar irregularidades do registro das informações de óbitos. Para o seu cálculo, foram utilizadas as informações de óbitos infantis correspondentes aos anos de 1996, 1997 e 1998 . Valores maiores do que $20 \%$ foram considerados excessivos.

A partir desses cinco indicadores, foram estabelecidos critérios para qualificar as informações de óbitos e nascimentos. Essas foram consideradas adequadas quando satisfaziam aos seguintes critérios: CGM padronizado por idade maior do que 6,75 por 1.000 habitantes, taxa de natalidade maior do que 16 por 1.000 habitantes, desvio médio do CGM menor do que $10 \%$, desvio médio da taxa de natalidade menor do que $10 \%$ e proporção de óbitos maldefinidos menor do que $20 \%$.

Foi realizada, então, a apreciação dos cinco indicadores em cada um dos municípios, classificando-os segundo a adequação das informações do SIM e do SINASC, em três distintas categorias:

1) Adequado nas informações de nascimentos e óbitos - satisfaz a todos os critérios;

2) Não satisfaz a algum dos critérios, mas apresenta CGM padronizado maior ou igual a 4 por 1.000 habitantes;

3) Grande deficiência nas informações de óbitos - quando não há informação de óbitos para grande parte da população, indicado pelo CGM padronizado menor do que 4 por 1.000 habitantes.

Para avaliar a adequação das informações disponíveis por UF, foram calculados o percentual de municípios e a correspondente proporção da população total em cada uma das categorias propostas. Um índice sintetizador, denominado de "índice de adequação" foi construído da seguinte forma:

Índice de adequação $=\mathrm{PI}+1 / 2 \mathrm{PII}-\mathrm{PIII}$ onde,
PI = proporção da população da UF classificada na Categoria I;

$\mathrm{PII}=$ proporção da população da UF classi ficada na Categoria II;

PIII = proporção da população da UF classificada na Categoria III.

É importante destacar que os critérios aqui utilizados para estabelecer a adequação da informação dos óbitos e nascimentos se prestam, principalmente, para apontar deficiências. Não conseguem, todavia, estabelecer se as coberturas dos sistemas de informação são, de fato, completas. Nesse sentido, foi necessária a aplicação de um modelo demográfico para a comparação com os resultados obtidos de forma direta. Optou-se, nesta análise, por usar uma modificação do modelo de ajuste de tábuas de vida da Organização das Nações Unidas - ONU (UN, 1982), conforme proposto por Szwarcwald \& Castilho (1995), que possibilita sua aplicação em populações com estatísticas vitais incompletas. Para constituir o modelo médio brasileiro, foram agregados todos os municípios pertencentes às sete UF onde, consensualmente, as informações vitais são fidedignas (IDB-2000) e classificados na Categoria I em relação à adequação das informações. Nesse agrupamento, considerou-se que a mortalidade infantil poderia ser calculada pelo método direto, utilizando-se o vetor constituído dos logitos das probabilidades de morte por idade como o vetor-padrão.

Os municípios foram agregados em estratos, por Grande Região, de acordo com a categoria de adequação das informações disponíveis. Em cada um dos estratos regionais compostos por municípios classificados na Categoria I, foi aplicado o modelo da ONU.

Tendo em vista que os municípios brasileiros que satisfaziam a todos os critérios propostos demonstraram também elevada cobertura das informações de óbitos, foi possível constituir um modelo médio nacional, formado a partir da agregação dos municípios classificados na Categoria I de todas as regiões do país, excluindo-se apenas o Distrito Federal, por ter suscitado incerteza em relação à estimativa obtida por cálculo direto. Em uma segunda etapa, foi aplicado o modelo da ONU para estimar a mortalidade infantil nos estratos regionais que demonstraram deficiências das informações do SIM e do SINASC, tomando-se como vetorpadrão o modelo médio nacional.

A mortalidade infantil por Grande Região foi estimada como média ponderada dos estratos regionais, considerando-se como ponderações os percentuais representativos da população menor de um ano, de cada estrato, na res- 
pectiva região. A mortalidade infantil para o Brasil foi estimada, por sua vez, como média ponderada das estimativas regionais, tendo como ponderações, desta feita, o número estimado de nascidos vivos para 1998.

\section{Inconsistências das estimativas do IBGE}

A apreciação das estimativas oficiais da mortalidade infantil no Brasil, fornecidas pelo IBGE, junto à análise das informações de nascimentos e óbitos dos sistemas do MS, mostra inconsistências importantes nas estimativas realizadas por mensuração indireta, que merecem ser destacadas.

Na Tabela 1, apresentam-se as estimativas das coberturas de óbitos infantis informados pelo SIM, obtidas pelo IBGE, para o período 1996-1998, por Grande Região. Observa-se aumento das coberturas nas regiões Norte e Nordeste. Porém, de acordo com essas estimativas, na Região Sudeste, a cobertura de óbitos infantis teria decrescido de $94 \%$, em 1996, para $81 \%$, em 1998. Tratando-se de uma região que tem apresentado, historicamente, regularidade das informações de registro, é difícil de acreditar que a subenumeração de óbitos tenha, de fato, aumentado de $6 \%$ para $19 \%$, nos três anos considerados.

Analisando, por sua vez, as estimativas das coberturas segundo as UF que compõem as regiões Sul e Sudeste, as incoerências são ainda mais acentuadas (Tabela 2). Por exemplo, a cobertura de óbitos infantis, estimada pelo IBGE, para o Estado do Rio de Janeiro, em 1997, é superior a $100 \%$ (de $110 \%$ ), mas decresce para $89 \%$, em 1998. Ou seja, segundo as estimativas do IBGE, a cobertura de óbitos infantis nesse estado teria diminuído em $20 \%$. Da mesma forma, em São Paulo, estado que, reconhecidamente, tem um dos melhores sistemas de informações do país, a cobertura estimada, em 1997, é de $92 \%$, porém decresce para $82 \%$, em 1998.

Observando as estimativas de cobertura de óbitos infantis para as demais UF, percebe-se outras incoerências (Tabela 2). A título de exemplificação, estado como Amapá, onde mais de $20 \%$ da população apresenta grande precariedade na informação de óbitos (ver seção seguinte), tem, de acordo com as estimativas do IBGE, cobertura superior ao Estado de São Paulo.

A análise que conjuga a estimativa do Estado do Amazonas e a estimativa no município da capital, para o ano de 1998, aponta também para incorreções nas estimativas por mensuração indireta. A taxa de mortalidade infantil em Manaus foi estimada em 38,4 por $1.000 \mathrm{NV}$, e no Estado do Amazonas em 33,1 por $1.000 \mathrm{NV}$, o que levaria a uma estimativa de 28,4 por 1.000 no interior do estado, evidentemente muito inferior à esperada para o padrão de desenvolvimento do interior do Estado do Amazonas.

A comparação das estimativas municipais, obtidas por mensuração indireta pelo IBGE com os coeficientes de mortalidade infantil calculados pelo método direto nas capitais brasileiras mostra, igualmente, disparidades relevantes (Tabela 3). Chama a atenção, por exemplo, a estimativa fornecida pelo IBGE para Recife. Se correta, a estimativa da mortalidade infantil, calculada em 38 por $1.000 \mathrm{NV}$, estaria indicando um sub-registro de óbitos infantis de mais de 70\% nesse município. De maneira similar, a estimativa da mortalidade infantil em Curitiba estaria apontando para uma cobertura de óbi-

Tabela 1

Número de óbitos em crianças menores de um ano de idade, informado no Sistema de Informações sobre Mortalidade (SIM) do Ministério da Saúde (MS), e coberturas estimadas pela Fundação Instituto Brasileiro de Geografia e Estatística (IBGE). Brasil, 1996-1998.

\begin{tabular}{|c|c|c|c|c|c|c|}
\hline \multirow[t]{2}{*}{ Região } & \multicolumn{3}{|c|}{ Número de óbitos (SIM/MS) } & \multicolumn{3}{|c|}{ Coberturas estimadas pelo IBGE } \\
\hline & 1996 & 1997 & 1998 & 1996 & 1997 & 1998 \\
\hline Norte & 5.989 & 6.203 & 6.741 & 47,85 & 49,68 & 57,05 \\
\hline Nordeste & 23.274 & 22.094 & 23.754 & 33,30 & 33,48 & 38,58 \\
\hline Sudeste & 31.945 & 30.133 & 27.599 & 93,51 & 89,36 & 80,72 \\
\hline Sul & 9.176 & 8.266 & 8.594 & 86,37 & 74,77 & 79,84 \\
\hline Centro-Oeste & 4.639 & 4.704 & 4.699 & 76,10 & 74,28 & 74,29 \\
\hline Brasil & 75.023 & 71.400 & 71.387 & 56,28 & 55,10 & 57,26 \\
\hline
\end{tabular}

Fonte: Simões (1999). 
Tabela 2

Coberturas (\%) dos óbitos infantis informados no Sistema de Informações sobre Mortalidade (SIM) do Ministério da Saúde (MS), e estimadas pela Fundação Instituto Brasileiro de Geografia e Estatística (IBGE) por Unidade da Federação. Brasil, 1997-1998.

\begin{tabular}{|c|c|c|}
\hline \multirow{2}{*}{$\begin{array}{l}\text { Unidade da } \\
\text { Federação }\end{array}$} & \multicolumn{2}{|c|}{ Cobertura (\%) } \\
\hline & 1997 & 1998 \\
\hline Rondônia & 63,6 & 67,4 \\
\hline Acre & 55,6 & 81,8 \\
\hline Amazonas & 57,4 & 64,2 \\
\hline Roraima & 47,2 & 56,5 \\
\hline Pará & 39,8 & 47,4 \\
\hline Amapá & 93,5 & 87,3 \\
\hline Tocantins & 49,8 & 55,1 \\
\hline Maranhão & 13,6 & 20,3 \\
\hline Piauí & 17,1 & 20,4 \\
\hline Ceará & 40,4 & 40,0 \\
\hline Rio Grande do Norte & 33,8 & 36,7 \\
\hline Paraíba & 30,1 & 23,8 \\
\hline Pernambuco & 52,0 & 52,0 \\
\hline Alagoas & 31,2 & 55,8 \\
\hline Sergipe & 47,2 & 62,0 \\
\hline Bahia & 31,1 & 37,1 \\
\hline Minas Gerais & 75,5 & 73,6 \\
\hline Espírito Santo & 69,0 & 74,5 \\
\hline Rio de Janeiro & 109,7 & 89,3 \\
\hline São Paulo & 92,4 & 81,9 \\
\hline Paraná & 69,4 & 76,8 \\
\hline Santa Catarina & 74,4 & 74,0 \\
\hline Rio Grande do Sul & 83,4 & 87,9 \\
\hline Mato Grosso do Sul & 97,7 & 98,6 \\
\hline Mato Grosso & 58,1 & 64,4 \\
\hline Goiás & 70,7 & 71,3 \\
\hline Distrito Federal & 82,4 & 72,2 \\
\hline Brasil & 55,1 & 57,3 \\
\hline
\end{tabular}

Fonte: DATASUS (2001).
Tabela 3

Coeficientes de mortalidade infantil calculados pelo método direto e estimados pela Fundação Instituto Brasileiro de Geografia e Estatística (IBGE) nos municípios das capitais. Brasil, 1998.

\begin{tabular}{|c|c|c|}
\hline \multirow[t]{2}{*}{ Município } & \multicolumn{2}{|c|}{$\begin{array}{c}\text { Mortalidade infantil } \\
\text { (por } 1.000 \text { nascidos vivos) }\end{array}$} \\
\hline & $\begin{array}{l}\text { Método } \\
\text { direto } 1\end{array}$ & $\begin{array}{c}\text { Estimada } \\
\text { pelo IBGE2 }\end{array}$ \\
\hline Porto Velho & 31,9 & 26,1 \\
\hline Rio Branco & 31,3 & 46,0 \\
\hline Manaus & 35,1 & 38,4 \\
\hline Boa Vista & 19,2 & 29,8 \\
\hline Belém & 30,4 & 29,8 \\
\hline Macapá & 24,1 & 25,2 \\
\hline Palmas & 24,2 & 28,8 \\
\hline São Luís & 22,7 & 45,5 \\
\hline Teresina & 18,0 & 30,3 \\
\hline Fortaleza & 31,1 & 51,1 \\
\hline Natal & 17,9 & 44,7 \\
\hline João Pessoa & 16,8 & 46,3 \\
\hline Recife & 21,6 & 38,0 \\
\hline Maceió & 47,2 & 53,2 \\
\hline Aracaju & 28,9 & 42,9 \\
\hline Salvador & 25,7 & 31,3 \\
\hline Belo Horizonte & 20,6 & 30,4 \\
\hline Vitória & 15,0 & 25,6 \\
\hline Rio de Janeiro & 18,2 & 24,8 \\
\hline São Paulo & 18,8 & 20,9 \\
\hline Curitiba & 16,6 & 22,5 \\
\hline Florianópolis & 13,9 & 19,7 \\
\hline Porto Alegre & 16,4 & 21,3 \\
\hline Campo Grande & 18,8 & 19,3 \\
\hline Cuiabá & 21,0 & 21,6 \\
\hline Goiânia & 19,9 & 24,3 \\
\hline Brasília & 16,4 & 23,2 \\
\hline
\end{tabular}

Fonte: 1 DATASUS (2001); 2 Simões (1999). tos infantis de apenas $74 \%$, menor do que a encontrada nos Estados do Amapá e Acre.

É importante destacar que muitas dessas cidades têm regularidade das informações de registro de óbitos e são predominantemente urbanas. As grandes proporções de sub-registro, estimadas com valores maiores do que $20 \%$ em grande parte dos municípios analisados, sugerem falhas nos procedimentos metodológicos utilizados para obtenção das estimativas por parte do IBGE, e apontam que a mortalidade infantil deve ser calculada pelo método direto, nos locais onde houver adequação das informações de nascimento e óbitos.

\section{O que dizem as informações de óbitos e nascimentos do Ministério da Saúde}

Após a análise das fontes de informações de nascimentos e óbitos do MS, os municípios de cada UF foram agregados segundo a categoria de adequação das informações (Tabela 4). Verifica-se que a Região Norte é a que possui as maiores deficiências na cobertura das informações de óbitos. Do total de municípios da região, $63 \%$ têm registro inadequado de óbitos, constituindo, aproximadamente, $35 \%$ da população total. A Região Nordeste, embora com situação um pouco melhor, também revela grande precariedade das informações de óbitos. Do total de municípios da região, 53\% apresentam 
Tabela 4

Proporção de municípios e proporção da população total de acordo com a categoria de adequação das informações vitais, segundo as Unidades da Federação e Grande Região. Brasil, 1998.

\begin{tabular}{|c|c|c|c|c|c|c|c|}
\hline \multirow{2}{*}{$\begin{array}{l}\text { Unidade da Federação } \\
\text { e Região }\end{array}$} & \multicolumn{2}{|c|}{ Categoria I } & \multicolumn{2}{|c|}{ Categoria II } & \multicolumn{2}{|c|}{ Categoria III } & \multirow{2}{*}{$\begin{array}{l}\text { Índice de } \\
\text { adequação }\end{array}$} \\
\hline & $\begin{array}{l}\text { Proporção } \\
\text { da população } \\
\text { total (\%) }\end{array}$ & $\begin{array}{l}\text { Proporção de } \\
\text { municípios (\%) }\end{array}$ & $\begin{array}{l}\text { Proporção } \\
\text { da população } \\
\text { total (\%) }\end{array}$ & $\begin{array}{l}\text { Proporção de } \\
\text { municípios (\%) }\end{array}$ & $\begin{array}{l}\text { Proporção } \\
\text { da população } \\
\text { total (\%) }\end{array}$ & $\begin{array}{l}\text { Proporção de } \\
\text { municípios (\%) }\end{array}$ & \\
\hline Rondônia & 11,1 & 3,9 & 73,2 & 57,7 & 15,7 & 38,5 & 32,0 \\
\hline Acre & 48,6 & 4,6 & 38,4 & 45,5 & 13,0 & 50,0 & 54,8 \\
\hline Amazonas & 48,6 & 1,6 & 9,7 & 14,5 & 41,8 & 83,9 & 11,7 \\
\hline Roraima & 62,5 & 6,7 & 17,0 & 40,0 & 20,5 & 53,3 & 50,5 \\
\hline Pará & 27,7 & 2,1 & 31,7 & 31,5 & 40,6 & 66,4 & 3,0 \\
\hline Amapá & 58,2 & 6,3 & 19,3 & 12,5 & 22,5 & 81,3 & 45,4 \\
\hline Tocantins & 10,1 & 0,7 & 55,6 & 40,3 & 34,3 & 59,0 & 3,6 \\
\hline Norte & 31,5 & 2,2 & 33,3 & 35,2 & 35,3 & 62,6 & 12,9 \\
\hline Maranhão & 15,3 & 0,5 & 27,8 & 15,7 & 56,9 & 83,9 & $-27,7$ \\
\hline Piauí & 0,0 & 0,0 & 53,6 & 18,6 & 46,4 & 81,5 & $-19,6$ \\
\hline Ceará & 29,3 & 0,5 & 39,5 & 50,0 & 31,2 & 49,5 & 17,9 \\
\hline Rio Grande do Norte & 2,0 & 0,6 & 75,7 & 50,0 & 22,3 & 49,4 & 17,6 \\
\hline Paraíba & 0,0 & 0,0 & 62,2 & 41,7 & 37,8 & 58,3 & $-6,7$ \\
\hline Pernambuco & 32,3 & 6,0 & 65,0 & 85,4 & 2,7 & 8,7 & 62,1 \\
\hline Alagoas & 2,8 & 3,0 & 90,7 & 79,2 & 6,5 & 17,8 & 41,7 \\
\hline Sergipe & 33,2 & 4,0 & 51,1 & 65,3 & 15,7 & 30,7 & 43,1 \\
\hline Bahia & 23,3 & 2,2 & 44,5 & 42,4 & 32,2 & 55,4 & 13,4 \\
\hline Nordeste & 19,6 & 1,6 & 51,7 & 45,1 & 28,7 & 53,3 & 16,8 \\
\hline Minas Gerais & 28,3 & 4,6 & 63,9 & 73,9 & 7,8 & 21,6 & 52,5 \\
\hline Espírito Santo & 50,2 & 11,7 & 48,3 & 81,8 & 1,6 & 6,5 & 72,8 \\
\hline Rio de Janeiro & 88,2 & 50,6 & 11,8 & 49,5 & 0,0 & 0,0 & 94,1 \\
\hline São Paulo & 76,4 & 31,5 & 23,5 & 66,5 & 0,1 & 2,0 & 88,1 \\
\hline Sudeste & 65,7 & 17,8 & 32,3 & 70,1 & 2,0 & 12,1 & 79,9 \\
\hline Paraná & 52,6 & 23,3 & 46,7 & 73,4 & 0,6 & 3,3 & 75,4 \\
\hline Santa Catarina & 42,9 & 13,0 & 54,9 & 75,8 & 2,2 & 11,3 & 68,2 \\
\hline Rio Grande do Sul & 64,3 & 17,1 & 34,4 & 73,5 & 1,4 & 9,4 & 80,1 \\
\hline Sul & 55,4 & 18,2 & 43,4 & 74,0 & 1,3 & 7,8 & 75,8 \\
\hline Mato Grosso do Sul & 61,1 & 23,4 & 37,1 & 66,2 & 1,8 & 10,4 & 77,9 \\
\hline Mato Grosso & 30,0 & 8,7 & 59,6 & 63,5 & 10,5 & 27,8 & 49,3 \\
\hline Goiás & 45,0 & 6,2 & 50,1 & 70,3 & 4,9 & 23,6 & 65,2 \\
\hline Distrito Federal & 100,0 & 100,0 & 0,0 & 0,0 & 0,0 & 0,0 & 100,0 \\
\hline Centro-Oeste & 54,4 & 10,1 & 41,0 & 67,5 & 4,7 & 22,4 & 70,2 \\
\hline Brasil & 47,8 & 10,7 & 40,1 & 59,7 & 12,1 & 29,5 & 55,8 \\
\hline
\end{tabular}

Fonte: Dados primários; DATASUS (2001). 
coeficiente geral de mortalidade padronizado por idade menor do que 4, o que representa 29\% da população da região.

De acordo com o percentual da população que reside em municípios classificados na Categoria III, os resultados mostram ainda que a região que melhor informa a mortalidade é a Região Sul, onde somente $1 \%$ da população apresenta grande precariedade dos dados de óbitos. Igualmente, nas regiões Sudeste e Centro-Oeste, um número bem reduzido de municípios não notifica a mortalidade, em proporção menor do que 5\% da população total. Para a totalidade do Brasil, $12 \%$ da população vive em locais com grandes deficiências na informação da mortalidade.

No que diz respeito à apreciação dos resultados por UF, os estados do Amazonas e Pará, na Região Norte, e Maranhão e Piauí, no Nordeste, merecem atenção específica, com mais de $40 \%$ da população classificada na Categoria III. No Maranhão, 57\% da população apresenta grande deficiência na cobertura das informações de mortalidade.

No tocante aos resultados relativos ao índice de adequação por UF, observa-se que alguns Estados da Região Norte, como Acre, Roraima e Amapá, apresentam valores do índice próximos a 50\%. Expressando a concentração da população nas categorias extremas (I e III), os valores medianos encontrados são explicados pela grande proporção da população desses Estados que reside nos municípios das capitais. Em segundo lugar, os valores negativos encontrados no Maranhão, Piauí e Paraíba indicam que a parcela da população que tem precariedade da informação de mortalidade nesses Estados é maior do que a parcela da população que tem notificação satisfatória. Por outro lado, o valor de $62 \%$ em Pernambuco, sugere que esse é o Estado nordestino que tem melhor registro das estatísticas vitais.

Panorama bem distinto é encontrado nas regiões Sul e Sudeste. Os Estados dessas regiões, na sua maioria, têm índices maiores do que $70 \%$, o que poderia, portanto, constituir um limite crítico inferior para o estabelecimento de adequação satisfatória. No Sudeste, o único Estado que mostra falhas nas estatísticas vitais é Minas Gerais, sobretudo nas informações do SINASC.

Na Região Centro-Oeste, valores do índice de adequação sugerem que as coberturas do SIM nos Estados do Mato Grosso e Goiás ainda não são completas. Apreciação específica merece o Distrito Federal, que foi classificado na Categoria I. Embora apresente um dos melhores conjuntos de indicadores de adequação das informações do Brasil, com extrema regularidade temporal tanto das informações de óbitos como de nascimentos, Brasília apresenta taxa de natalidade de 25 por 1.000 habitantes, que é excessivamente alta para o padrão regional, sugerindo uma provável importação de nascimentos de municípios vizinhos.

As estimativas da mortalidade infantil por aplicação do modelo da ONU a cada estrato regional, composto por municípios classificados na Categoria I, isto é, considerados com qualidade adequada das informações do SIM e do SINASC, estão apresentadas na Tabela 5. Comparando-se os valores estimados da mortalidade infantil com os observados, verifica-se que as razões entre as estimativas foram maiores do que $90 \%$ em todos os estratos regionais. A menor razão entre as taxas foi encontrada para Brasília, de $91 \%$. O segundo menor valor foi encontrado para o estrato formado por municípios do Nordeste, mas igual a $95 \%$, aproximadamente.

Os resultados obtidos pela aplicação do modelo da ONU aos demais estratos regionais, tomando-se como vetor-padrão o modelo médio nacional, estão dispostos na Tabela 6. A cobertura muito baixa no estrato da Região Nordeste, composto por municípios classificados na Categoria III, que varia com a idade, dificulta a aplicação do modelo demográfico. Sob diferentes estimativas do sub-registro no grupo etário de 0-4 anos, a estimativa varia, neste estrato, de 74 para 82 por 1.000 NV. É interessante observar que nos estratos compostos por municípios das regiões Sudeste e Sul, as estimativas obtidas pelo modelo demográfico são bem próximas às calculadas pelo método direto. Diferença maior do que a esperada, de 3 por $1.000 \mathrm{NV}$, foi encontrada para o estrato formado pelos municípios do Mato Grosso do Sul classificados na Categoria II.

A mortalidade infantil por Grande Região foi estimada como média ponderada dos estratos regionais. São fornecidas duas estimativas na Tabela 7. Para obtenção da primeira, denominada de valor inferior, corrigiram-se os óbitos informados em menores de um ano apenas nos estratos compostos por municípios que não satisfizeram a pelo menos um dos critérios propostos, isto é, os compostos por municípios classificados nas categorias II e III. Nos estratos regionais formados por municípios classificados na Categoria I e nos sete Estados, Espírito Santo, Rio de Janeiro, São Paulo, Paraná, Santa Catarina, Rio Grande do Sul e Mato Grosso do Sul, a mortalidade infantil foi calculada pelo método direto. Adicionalmente, foram utilizados os valores inferiores das estimativas da mor- 
Estimativas da mortalidade infantil por aplicação do modelo da Organização das Nações Unidas (ONU) nos estratos compostos por municípios classificados na Categoria I. Brasil, 1998.

\begin{tabular}{|c|c|c|c|}
\hline \multirow[t]{2}{*}{ Estrato } & \multicolumn{2}{|c|}{ Mortalidade infantil } & \multirow[t]{2}{*}{ Razão entre as estimativas } \\
\hline & Modelo da ONU & Cálculo direto & \\
\hline Norte I & 30,6 & 29,6 & 96,7 \\
\hline Nordeste I & 28,7 & 27,3 & 95,1 \\
\hline Minas Gerais I & 23,6 & 23,3 & 98,7 \\
\hline Mato Grosso e Goiás I & 20,0 & 19,9 & 99,5 \\
\hline Distrito Federal I & 18,1 & 16,4 & 90,6 \\
\hline
\end{tabular}

Tabela 6

Estimativas da mortalidade infantil nos estratos considerados segundo o método utilizado. Brasil, 1998.

\begin{tabular}{|c|c|c|c|c|}
\hline \multirow[t]{3}{*}{ Estrato } & \multicolumn{4}{|c|}{ Estimativas da mortalidade infantil } \\
\hline & \multicolumn{2}{|c|}{ Valor superior } & \multicolumn{2}{|c|}{ Valor inferior } \\
\hline & Método* & Estimativa & Método* & Estimativa \\
\hline Região Norte I & 2 & 30,6 & 1 & 29,6 \\
\hline Região Norte II & 2 & 33,8 & 2 & 33,8 \\
\hline Região Norte III & 2 & 42,0 & 2 & 42,0 \\
\hline Região Nordeste I & 2 & 28,7 & 1 & 27,3 \\
\hline Região Nordeste II & 4 & 41,2 & 3 & 37,5 \\
\hline Região Nordeste III & 4 & 81,9 & 3 & 74,4 \\
\hline Espírito Santo, Rio de Janeiro e São Paulo I & 2 & 21,7 & 1 & 20,5 \\
\hline Espírito Santo, Rio de Janeiro e São Paulo II & 2 & 20,4 & 1 & 19,5 \\
\hline Minas Gerais I & 2 & 23,6 & 1 & 23,3 \\
\hline Minas Gerais II & 2 & 22,2 & 2 & 22,2 \\
\hline Região Sul I & 2 & 19,4 & 1 & 19,1 \\
\hline Região Sul II & 2 & 18,6 & 1 & 18,2 \\
\hline Mato Grosso do Sul I & 2 & 23,8 & 1 & 23,6 \\
\hline Mato Grosso do Sul II & 2 & 31,1 & 1 & 28,0 \\
\hline Mato Grosso e Goiás I & 2 & 20,0 & 1 & 19,9 \\
\hline Mato Grosso e Goiás II & 2 & 24,7 & 2 & 24,7 \\
\hline Distrito Federal I & 2 & 18,1 & 2 & 18,1 \\
\hline
\end{tabular}

* Métodos: (1) Cálculo direto; (2) Modelo da Organização das Nações Unidas (ONU) - sub-registro constante para 1 ano e mais de idade; (3) Modelo da ONU - sub-registro diferenciado por grupo etário ( $<1$ ano, 1-4 anos, 5 anos e mais); cálculo baseado nas estimativas de sub-registro encontradas nos grupos $0-4$ anos e 5 anos e mais; (4) Modelo da ONU - sub-registro diferenciado por grupo etário (< 1 ano, 1-4 anos, 5 anos e mais); cálculo baseado nas estimativas de sub-registros encontradas nos grupos 1-4 anos e 5 anos e mais.

talidade infantil obtidas pelo modelo da ONU nos estratos da Região Nordeste. Já para obtenção do limite superior da estimativa, a mortalidade infantil foi ajustada pelo modelo da ONU em todos os estratos regionais, além de se considerar os valores superiores da estimativa da mortalidade infantil nos estratos da Região Nordeste.

A mortalidade infantil para o Brasil foi estimada, por sua vez, como média ponderada das estimativas regionais. Utilizando esse procedimento metodológico, a mortalidade infantil para o Brasil foi estimada dentro do intervalo 30,7-32,6 por $1.000 \mathrm{NV}$ (Tabela 7 ).

Em relação à cobertura das informações de óbitos infantis, a região que demonstra o maior sub-registro é a Nordeste, seguindo-se da Região Norte. Na totalidade do Brasil, a estimativa da cobertura de óbitos infantis variou de $61,8 \%$ a $65,6 \%$ (Tabela 7 ). 
Estimativas da mortalidade infantil e da cobertura de óbitos infantis por Grande Região. Brasil, 1998.

\begin{tabular}{|c|c|c|c|c|}
\hline \multirow[t]{2}{*}{ Região } & \multicolumn{2}{|c|}{ Mortalidade infantil } & \multicolumn{2}{|c|}{ Cobertura de óbitos infantis (\%) } \\
\hline & Valor inferior & Valor superior & Valor superior & Valor inferior \\
\hline Norte & 35,9 & 36,2 & 53,3 & 52,9 \\
\hline Nordeste & 47,4 & 51,9 & 44,1 & 40,3 \\
\hline Sudeste & 20,8 & 21,7 & 96,9 & 92,9 \\
\hline Sul & 18,7 & 19,0 & 100,0 & 98,5 \\
\hline Centro-Oeste & 22,6 & 22,8 & 88,5 & 87,5 \\
\hline Brasil & 30,7 & 32,6 & 65,6 & 61,8 \\
\hline
\end{tabular}

\section{Discussão}

No Brasil, o desconhecimento sobre os limites dos dados disponíveis para o cálculo da mortalidade infantil, levando ao uso de procedimentos de mensuração indireta, indistintamente, em qualquer que seja a área geográfica, tem resultado em superestimativas, que têm tido reflexo negativo tanto no plano nacional (ABRASCO, 2001) como no plano internacional. Vale lembrar que incorreções nas estimativas oficiais da mortalidade infantil e da cobertura de óbitos informados conduzem a erros na estimação de outros indicadores, como, por exemplo, a esperança de vida saudável (HALE), estimada pela Organização Mundial da Saúde (OMS) nos relatórios de 2000 e 2001 (WHO, 2000, 2001), com base na estimativa da tábua de vida brasileira fornecida pelo IBGE.

Adicionalmente, os achados deste trabalho apontaram para inconsistências relevantes nas estimativas obtidas por técnicas de mensuração indireta pelo IBGE, tanto nas estimativas da mortalidade infantil como nas da cobertura de óbitos informados. Constatou-se também, que quanto menor o nível de agregação geográfica, maior é o erro na estimativa obtida por mensuração indireta, como no caso das estimativas fornecidas por município (Simões, 1999).

O SIM vem demonstrando nítidos avanços, seja no que se refere à ampliação da cobertura, seja na divulgação dos dados. A oportunidade de examinar as informações de óbitos e nascimentos em todos os municípios brasileiros, abriu novas possibilidades para examinar a qualidade da informação e identificar irregularidades locais, passíveis de melhora com o tempo. Dos resultados aqui apresentados, depreendeu-se que em, pelo menos $50 \%$ da população, a cobertura das informações vitais pode ser considerada adequada. Para essa parcela da população, a melhor estimativa da mortalidade in- fantil é, obviamente, fornecida pelo cálculo direto.

Os problemas estão, justamente, em identificar as áreas onde a cobertura pode ser considerada completa. Os critérios propostos neste trabalho funcionaram como marcadores importantes da qualidade e regularidade da informação. Porém, esses critérios são, claramente, mais excludentes do que qualificadores da cobertura de óbitos informados, no sentido de que identificam bem os locais com cobertura adequada, mas não conseguem captar grande parte dos municípios que, reconhecidamente, têm cobertura completa das informações do SIM e do SINASC. Por outro lado, funcionam igualmente bem para identificar municípios com grande precariedade de informação. As maiores dificuldades, portanto, recaem nos municípios classificados na Categoria II.

Embora se tenha tentado superar a questão com a elaboração de um "índice de adequação", levando em consideração a parcela da população classificada na Categoria II ponderada por $50 \%$, valores mais altos no Rio de Janeiro e em São Paulo do que nos estados da Região Sul, estão expressando, mais provavelmente, as características demográficas desses estados do que propriamente a adequação da informação. A questão refere-se, justamente, a estabelecer valores críticos nacionais para os indicadores utilizados, tendo em vista a diversidade dos padrões regionais. Além disso, a diversidade dos padrões não se restringe às diferenças regionais, mas também se reproduz na análise dos dados por tamanho populacional do município ou situação urbano/rural.

Entende-se, portanto, que os critérios aqui estabelecidos precisam ser aperfeiçoados. Todavia, apesar das suas limitações, a análise das informações no nível de município permitiu identificar áreas com claras irregularidades de registro, assim como identificar municípios do 
Norte, Nordeste e Centro-Oeste para os quais é possível calcular a mortalidade infantil pelo método direto. Por outro lado, a análise da regularidade temporal das informações chama a atenção para a ampliação da cobertura dos sistemas de informação em diversas áreas do país. No que se refere ao SINASC, a cobertura tem aumentado, nitidamente, e no que diz respeito ao SIM, vários municípios já têm incorporado as informações provenientes do Sistema de Informações de Ações Básicas (SIAB). Sendo assim, pressupostos de sub-registros constantes no tempo não podem (e não devem) ser assumidos como verdadeiros.

Em relação ao método utilizado para corrigir a mortalidade infantil nos locais com deficiências na notificação das informações vitais, optou-se pelo modelo da ONU, por ter a vantagem de ajustar a curva completa da mortalidade por idade, e por ter sido utilizado, anteriormente, para ajustar esses dados no Brasil, com bons resultados (Szwarcwald \& Castilho, 1995). O procedimento consiste em ajustar a curva de mortalidade por idade a um modelo-padrão por intermédio da estimação de dois parâmetros de uma regressão linear que expressam, respectivamente, o nível de mortalidade e a relação entre a mortalidade na infância e a adulta. A partir de pressupostos sobre o comportamento do sub-registro de óbitos por idade, foi possível a sua aplicação para os estratos regionais com precariedade das informações vitais.

A maior fonte de erro nas estimativas obtidas por esse procedimento está na escolha do vetor-padrão. Na presente análise, a consistên- cia das estimativas obtidas por aplicação do modelo da ONU, quando comparadas às obtidas pelo método direto nos locais com cobertura satisfatória das estatísticas vitais, sugere que o vetor-padrão foi adequado. Variações maiores foram provenientes dos pressupostos a cerca do comportamento do sub-registro de óbitos por idade, na Região Nordeste, onde a hipótese de sub-registro constante por idade foi rejeitada. No estrato composto por municípios localizados nessa região e classificados na Categoria III, a estimativa variou de 74 a 82 por $1.000 \mathrm{NV}$, dependendo da suposição assumida.

É importante destacar que nos locais onde a precariedade das informações de registro é muito grande, outros métodos baseados em pesquisas locais são mais adequados. Nesse sentido, o MS tem dirigido esforços para integrar as informações do SIM e do SIAB, e para a realização de pesquisas de busca ativa de óbitos nos locais com grandes deficiências de registro.

Tendo em vista que por mais robustos que sejam os métodos utilizados, erros provenientes de pressupostos não verdadeiros, de modelagem matemática e de amostragem conduzem a variações consideráveis nas estimativas resultantes, conclui-se, este trabalho, relevando-se, uma vez mais, a importância da análise continuada das informações de nascimentos e óbitos, no sentido de contribuir à melhoria dos nossos sistemas de informação, para que, em futuro próximo, a mortalidade infantil possa ser avaliada de forma direta em todo o território nacional.

\section{Agradecimentos}

Este trabalho teve apoio financeiro do Conselho Nacional de Desenvolvimento Científico e Tecnológico (no 521116/97-0) e do Centro Nacional de Epidemiologia, Fundação Nacional de Saúde, Ministério da Saúde. 


\section{Referências}

ABRASCO (Associação Brasileira de Pós-Graduação em Saúde Coletiva), 2001. Pesquisa em saúde no Brasil: Urgência do debate. Boletim da ABRASCO, 79:1-2.

BECKER, R. A., 1991. Análise da Mortalidade. Delineamentos Básicos. Brasília: Ministério da Saúde. (mimeo.)

DATASUS (Departamento de Informática do SUS), 2001. Informações de Saúde. <http://www.datasus. gov.br>.

IBGE (Fundação Instituto Brasileiro de Geografia e Estatística), 1998. Estatísticas do Registro Civil, 1996. v. 23. Rio de Janeiro: IBGE.

IBGE (Fundação Instituto Brasileiro de Geografia e Estatística), 2001. População. Indicadores Sociais. <http://www.ibge.gov.br>.

KAHN, H. A. \& SEMPOS, C. T., 1989. Statistical Methods in Epidemiology. New York: Oxford University Press.

MELLO-JORGE, M. H. P., 1983. Sub-registro dos eventos vitais. Revista de Saúde Pública, 17:148-151.

MELLO-JORGE, M. H. P., 1987. Sub-registro de Óbitos e Aspectos da Legislação sobre Mortalidade. II Reunião Nacional do Subsistema de Informação sobre Mortalidade. Brasília: Ministério da Saúde. (mimeo.)

MELLO-JORGE, M. H. P.; GOTLIEB, S. L. D. \& OLIVEIRA, H., 1996. O Sistema de Informação sobre Nascidos Vivos: Primeira avaliação dos dados brasileiros. Informe Epidemiológico do SUS, 2:15-48.
MS (Ministério da Saúde), 1982. O Subsistema de Informações sobre Mortalidade. In: Estatísticas de Mortalidade: Brasil, 1979 (Ministério da Saúde, org.), pp. 2-56, Brasília: Centro de Documentação, MS.

SCHRAMM, J. M. A. \& SZWARCWALD, C. L., 2000. Sistema hospitalar como fonte de informações para estimar a mortalidade neonatal e a natimortalidade. Revista de Saúde Pública, 34:272-279.

SIMÕES, C. C., 1999. Brasil: Estimativas da Mortalidade Infantil por Microregiões e Municípios. Brasília: Secretaria de Políticas da Saúde, Secretaria Executiva, Ministério da Saúde.

SZWARCWALD, C. L. \& CASTILHO, E. A., 1995. Estimativas da mortalidade infantil no Brasil, década de oitenta: Proposta de procedimento metodológico. Revista de Saúde Pública, 29:451-462.

UN (United Nations), 1982. Model Life Tables for Developing Countries. Population Studies 77. New York: UN.

WHO (World Health Organization), 2000. The World Health Report, 2000. Geneva: WHO.

WHO (World Health Organization), 2001. The World Health Report, 2001. Geneva: WHO.

Recebido em 14 de novembro de 2001

Versão final reapresentada em 6 de maio de 2002

Aprovado em 17 de junho de 2002 